\title{
Pick-by-Vision: Potenziale in der Unterstützung der Kommissionierung durch Smart Glasses
}

Sebastian Werning, Dennis Konusch und Ingmar Ickerott

Der Einsatz von Smart Glasses in der Logistik gilt im Zeitalter der Industrie 4.0 als sehr umstritten. Die Digitalisierung von Logistikprozessen schreitet voran, jedoch werden Potenziale in solch innovativen Technologien selten erkannt. Zur Identifikation relevanter Erfolgsfaktoren und Anforderungen der Logistik an die Informationstechnik eines Unternehmens wurde eine theoretische und praxisbasierte Informationsgrundlage herangezogen. Mithilfe einer Anbieterrecherche, einer Umfrage zum Thema Gebrauchstauglichkeit von Smart Glasses und einer systematischen Literaturrecherche werden Erfolgsfaktoren zum Einsatz von solchen Datenbrillen in der Logistik identifiziert. Die Kommissionierung ist als einer der ressourcenintensivsten Prozesse in der Logistik definiert und wird daher als Haupteinsatzgebiet der Smart Glasses betrachtet. Mithilfe eines Bewertungsmodells können standardisierte- und Smart-Glasses-basierte Kommissioniermethoden verglichen werden. Die daraus resultierende Gegenüberstellung von Vor- und Nachteilen in Bezug auf drei unterschiedliche, betriebswirtschaftlich relevante Perspektiven ermöglicht eine Ableitung individueller Handlungsempfehlungen. Somit ermöglicht dieser Beitrag eine Bewertung des Potenzials der Smart-Glasses-basierten Kommissioniermethode im Rahmen prozessoptimierender Logistikmaßnahmen. Zum einen gilt dies für eine (a) strukturierte Gegenüberstellung des Potenzials der Smart-Glasses-basierten Kommissioniermethode mit bereits am Markt etablierten Kommissioniermethoden, zum anderen resultiert eine Ableitung von weiteren Forschungsbereichen zur Aufdeckung von Potenzialen prozessoptimierender Technologien in der Logistik. Als Implikation (b) für die Praxis werden ein Einordnungsrahmen und Handlungshinweise für Unternehmen für die Auswahl geeigneter Kommissioniermethoden zur Prozessoptimierung in der betriebsinternen Logistik vorgeschlagen.

\section{Einleitung}

Die Digitalisierung als eine der sog. Megatrends in der Logistik wird als Motivation herangezogen, um die Auswirkung neuer Technologien auf die zugehörige Branche zu analysieren (Klötzer und Pflaum 2017). Dieser Trend erreicht sowohl 
Großunternehmen als auch klein- und mittelständische Unternehmen. Das Zeitalter der Industrie 4.0 zeichnet sich durch eine wachsende Vernetzung von betriebswirtschaftlicher Infrastruktur aus und offenbart dabei eine Vielzahl an Digitalisierungs- und Verbesserungsmöglichkeiten. Dennoch birgt die Industrie $4.0 \mathrm{zu}-$ gleich diverse Herausforderungen für Unternehmen, welche oftmals mit kostenintensiven Prozessanpassungen verbunden sind (Elbestawi et al. 2018). Zur Integration von intelligenten Technologien und der Sicherung von Wettbewerbsvorteilen im Zuge des Industrie 4.0-Zeitalters bedarf es daher einer Identifikation und Berücksichtigung kritischer Erfolgsfaktoren.

Dieser Beitrag fokussiert die vielseitige Betrachtung der Eigenschaften von standardisierten Kommissioniermethoden gegenüber einem Smart-Glassesbasierten Assistenzsystem. Im Laufe der Untersuchungen wird die Smart-Glassesbasierte Kommissioniermethode auch als „Pick-by-Vision“ bezeichnet (Schwerdtfeger et al. 2009). Das Potenzial dieser Methode wird herausgearbeitet und mit alternativen Kommissioniermethoden verglichen.

Mithilfe eines Bewertungsmodells werden Vor- und Nachteile von Kommissioniermethoden beleuchtet sowie Einflussgrößen auf den betrachteten Prozess aufgedeckt. Dabei bezieht sich das Modell auf Aspekte wie Lager- und IT-Infrastrukturen und die Gebrauchstauglichkeit der eingesetzten Technologien. Das Ergebnis zeigt eine Klassifizierung der Anforderungen unterschiedlicher Kommissioniermethoden in der Logistik.

Um einen Mehrwert durch das Bewertungsmodell zu schaffen, wird im Zuge der Untersuchung auf theoretische- und praxisbasierte Informationsgrundlagen zurückgegriffen. Im darauffolgenden Abschnitt werden die Grundzüge der Logistik definiert. Die Kommissionierung wird dabei als einer der informationsintensivsten Logistikprozesse abgebildet und unterschiedliche Kommissioniermethoden näher beleuchtet. Anschließend wird ein Bewertungsmodell in Anlehnung an Picot und Reichwald (1984) angewendet, um die gesammelten Informationen aus drei verschiedenen Perspektiven zu beleuchten. Im letzten Abschnitt werden Handlungsempfehlungen abgeleitet und Ergebnisse diskutiert.

\section{Methodische Vorgehensweise}

Für die Sondierung etablierter Kommissioniermethoden, die zu einem StärkenSchwächen-Vergleich herangezogen werden können, wird eine systematische Literaturrecherche nach Webster and Watson (2002) durchgeführt. Diese Informationsgrundlage wird durch Ergebnisse von Labortests, Umfragen und Anbieterrecherchen ergänzt. Dabei handelt es sich bspw. um einen Labortest mit 29 Probanden, welche die Gebrauchstauglichkeit einer Smart Glasses bewerten sollen. Die Umfrage beinhaltet zwei Fragebögen bzgl. der Akzeptanz und Gebrauchstauglichkeit von Smart Glasses. Dabei handelt es sich um den „Unified Theory of Acceptance and Use of Technology“ (UTAUT)- und dem „System Usability Scale“ (SUS)-Fragebogen, die sich insb. mit IT-Artefakten beschäftigen, welche sich auf 
die oben genannten Themen, wie die Akzeptanz des Personals gegenüber neuen Technologien, beziehen. Ein modifiziertes Bewertungsmodell nach Picot und Reichwald (1984) betrachtet Kriterien aus diesen Fragebögen aus Sicht von drei betriebswirtschaftlich relevanten Perspektiven in Abschnitt 4.

\section{Definition: Logistik}

Laut Schulte umfasst die Definition des Begriffs „Logistik“ nicht nur die Materialflüsse, sondern sämtliche damit verbundenen Informationsflüsse. Seit etwa 1970 wird dieser Begriff grundsätzlich mit dem Lagern von Gütern in Verbindung gebracht, während heutzutage eine weitreichendere und schnell wachsende Bedeutung nachgewiesen werden kann (Schulte 2009). Folgende Prozesse können als Hauptprozesse der Logistik definiert werden: Wareneingang, Lagerung und Warenausgang (Arnold et al. 2007). Weiterhin sind die „vier R's der Logistik“ in der Literatur aufzufinden und stehen für das richtige Gut, welches im richtigen $\mathrm{Zu}$ stand, zur richtigen Zeit am richtigen Ort bereitzustellen ist (Pfohl 2018). Im folgenden Abschnitt werden die Kernprozesse der Distributionslogistik näher definiert.

\subsection{Kernprozesse der Distributionslogistik}

Im Folgendem werden die Kernprozesse der Distributionslogistik in Anlehnung an Hompel (2010) betrachtet, die den Wareneingang, die Einlagerung, die Kommissionierung und den Warenausgang umfassen (vgl. Abb. 1).

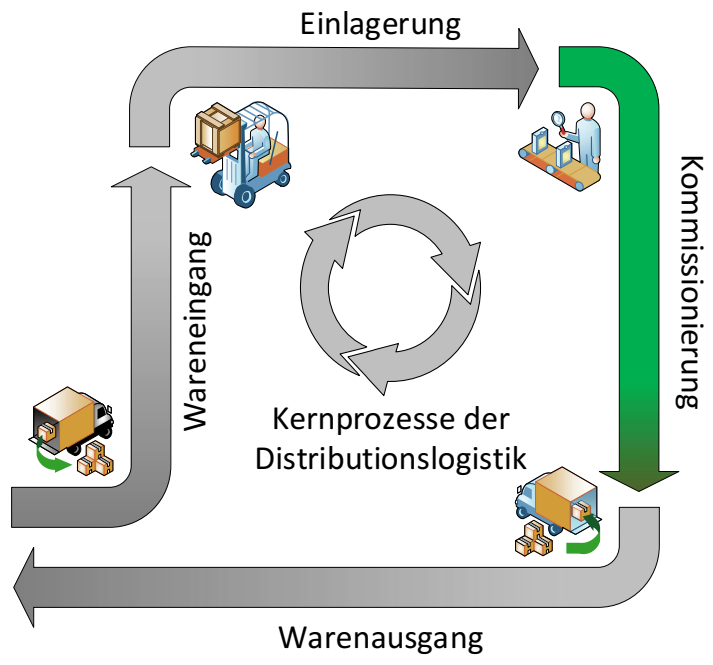

Abb. 1. Kernprozesse der Distributionslogistik, in Anlehnung an ten Hompel (2011) 
Ausgehend von einer Warenbestellung durch einen Disponenten starten die logistischen Prozesse in einem Unternehmen grundsätzlich mit einem Wareneingang. Präzise abgestimmte Liefertermine ermöglichen eine innerbetriebliche Vorbereitung und die Vermeidung von Wartezeiten (ten Hompel 2011). Zur Erbringung der Leistungsanforderung eines Wareneingangs müssen vereinheitlichte Ladungsträger genutzt und ausgelastet werden. Diese können zu beschleunigten Arbeitsprozessen in der Logistik führen (Arnold et al. 2007). Weiterhin können die Wareneingangsprüfung und die Bildung von Lagereinheiten als separate Vorgänge zwischen den Hauptprozessen interpretiert werden (ten Hompel 2011).

Die Einlagerung der Ware kann bspw. manuell durch Personaleinsatz und entsprechende Hilfsmittel wie Gabelstapler erfolgen. Dabei zielt die Einlagerung auf den innerbetrieblichen Transport von angelieferten Waren zu entsprechenden Lagervorrichtungen innerhalb eines Unternehmens ab (Martin 2014). Der Einlagerungsvorgang wird beendet, nachdem Mengen- und Qualitätskontrollen vollständig abgeschlossen sind.

Im Rahmen dieses Beitrags wird gezielt der Vorgang der Kommissionierung betrachtet, da sich der Kommissionierungsprozess zumeist als ein ressourcen- und informationsintensiver Prozess in Unternehmen herausstellt (Günther et al. 2009). Dadurch steckt in der Smart-Glasses-basierten Kommissionierung ein hohes Einsatzpotenzial. Der Kommissionierungsprozess wird gemäß der Richtlinie VDI 3590 als das Zusammenstellen bestimmter Teilmengen (Artikeln) auf Grund von Bedarfsinformationen aus einem Auftrag definiert (Bode und Preuß 2004). Jeder Griff zu einem Artikel, um diese auftragsorientiert zusammenzustellen, kann als „Pick“ bezeichnet werden. Im Kommissionierungsprozess geht es um die innerbetriebliche Fortbewegung der Güter, Organisation von angebrochenen Ladeeinheiten, ggf. der Verpackung von Entnahmemengen und der Bereitstellung der Ware am Warenausgang auf Basis eines bestimmten Auftrags (ten Hompel und Heidenblut 2008).

Der Warenausgangsprozess dient nach der Kommissionierung als Vorbereitung für den anschließenden Versand und kann durch Eigen- oder Fremdfuhrpark durchgeführt werden. Somit wird die Distribution der Ware an Kunden ermöglicht (Ehrmann 2017). Im Zuge der logistischen Geschäftsprozesse sind Rücknahmen auf Grund beschädigter Waren möglich, wodurch ein Rücklauf der entsprechenden Artikel zum Lieferanten in Gang gesetzt werden kann (Gudehus 2010).

\subsection{Definition: Kommissionierung}

Die Kommissionierung kann als ressourcenintensivster Prozess in der Logistik definiert werden. Grundsätzlich wird zwischen automatischer und manueller Kommissionierung unterschieden. Die automatische Kommissionierung kann über Kommissionierautomaten oder Kommissionierroboter erfolgen (Dekker et al. 2004). Die manuelle Kommissionierung umfasst hingegen die Kommissionierung durch einen Mitarbeiter. Bis zu 50 \% der Lagersysteme von Unternehmen greifen auf klassische „Mann-zu-Ware-Kommissionierungsmethoden“ zurück (Almeida 
und Ferreira 2009). Dabei handelt es sich um eine Kommissionierung, in der das Personal eingesetzt wird, um Waren zwischen zwei oder mehreren Orten zu transportieren.

Weiterhin kann zwischen ein- und mehrstufigen Kommissionierungsprozessen unterschieden werden. Die einstufige Kommissionierung umfasst eine auftragsorientierte Kommissionierung, bei der alle zu packenden Artikel eines Auftrags nacheinander zusammengestellt werden (Martin 2011). Die zwei- oder mehrstufige Kommissionierung hingegen beinhaltet eine artikelorientierte Arbeitsweise. In diesem Kommissionierungsprozess werden die Artikel nacheinander den jeweiligen Aufträgen zugeordnet, wodurch sich eine simultane Bearbeitung realisieren lässt (ten Hompel et al. 2011).

\subsection{Methoden der Kommissionierung}

Aufgrund der Vielzahl an Betriebs- und Lagerstrukturen von Unternehmen haben sich unterschiedliche Kommissioniermethoden entwickelt. In Anlehnung an Reif (2009) werden in fünf übliche Kommissioniermethoden dargestellt.

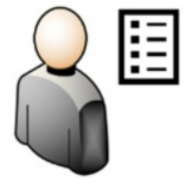

(a) Pick-by-Paper

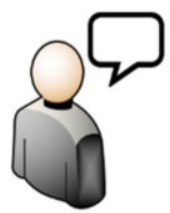

(c) Pick-by-Voice

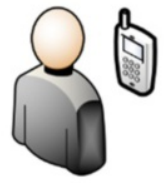

(b) Pick-by-Scan

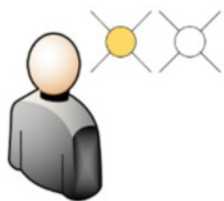

(d) Pick-by-Light

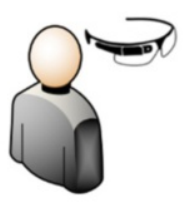

(e) Pick-by-Vision

Abb. 2. Etablierte Kommissioniermethoden, nach Reif (2009)

(a) Bei einer klassischen Kommissioniermethode handelt es sich um die Pickby-Paper-Kommissioniermethode. Hierbei arbeitet der Kommissionierer belegorientiert, entnimmt die benötigten Informationen einer Liste und sammelt die Ware in einer vorgegebenen Reihenfolge. Kommt es dabei zu Fehlern, werden diese auf der Liste notiert, sodass im Nachfolgeprozess entsprechend reagiert werden kann. In der Regel dient der Kommissionierauftrag als Grundlage für die Rechnungserstellung im Nachfolgeprozess (Arnold et al. 2008).

(b) Die Pick-by-Scan-Kommissioniermethode ersetzt die Liste durch ein mobiles Datenerfassungsgerät (MDE-Gerät). Hierbei handelt es sich um eine beleglose Kommissioniermethode. Im Sinne von Digitalisierungsmaßnahmen logistischer 
Prozesse nutzen Unternehmen die MDE-Geräte in Form eines Handhelds. Diese können sowohl offline- als auch online betrieben werden (Wölfle und Schubert 2006).

(c) Vor allem in den USA hat sich die Pick-by-Voice-Kommissioniermethode als weitere beleglose Vorgehensweise etabliert. Hierbei werden die Bedarfsinformationen akustisch mithilfe von Kopfhörern übermittelt, um einen Kommissionierauftrag mit freien Händen bearbeiten zu können.

(d) Die Pick-by-Light-Kommissioniermethode arbeitet mit am Regal angebrachten Lampen, die entsprechend des Auftrages an der Entnahmestelle für einen Kommissionierer aufleuchten. Somit findet die Übertragung der Informationen visuell am Platz des benötigten Artikels statt, sobald ein digitaler Auftrag eine bestimmte Auftragsposition anwählt (Gleißner und Femerling 2008).

(e) Besonders im Zuge der Industrie 4.0 werden innovative Technologien angewandt, um die Digitalisierung von Geschäftsprozessen zu ermöglichen. Das Sichern von Wettbewerbsvorteilen durch eine Verbesserung der Kommissionierleistung ist oftmals von entscheidender Bedeutung für die Wettbewerbspositionierung (Martin 2011). Unter innovativen Technologien fällt auch der Einsatz der Augmented Reality (AR). AR ergänzt die reale Welt mit der Einblendung von visuellen Objekten (Reif 2009). Im Rahmen der Kommissionierung wird die Technologie mit der Kommissioniermethode Pick-by-Vision (vgl. Abb. 3) verbunden und soll die Nutzung neuer Potenziale zur Verbesserung der Produktivität ermöglichen (Günthner et al. 2009).

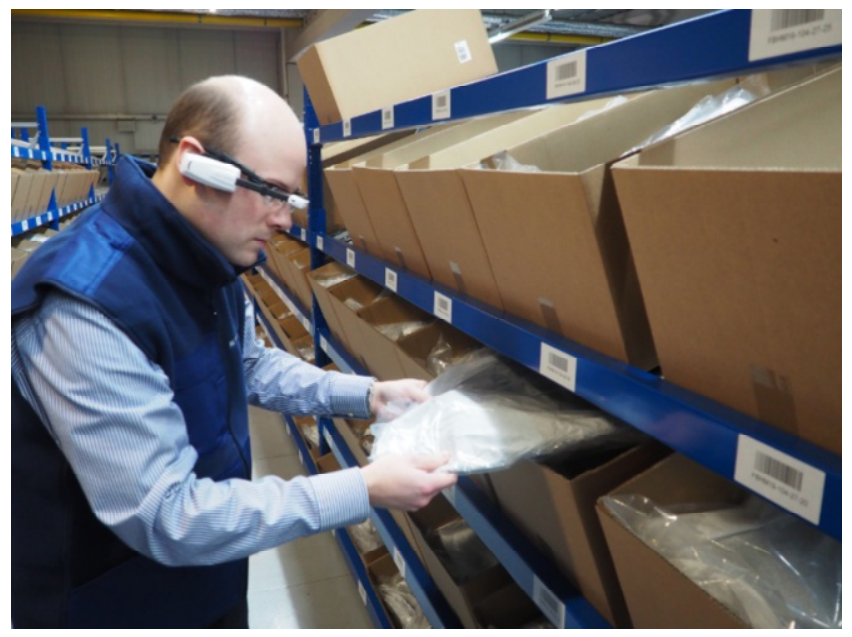

Abb. 3. Pick-by-Vision - Einsatz von Smart Glasses in der Kommissionierung

Informationen in Form von Texten und Bildern können direkt in das Sichtfeld des Nutzenden durch die Projektion auf ein integriertes Display angezeigt werden. Die AR-Technologie wird oftmals ebenfalls mit dem Begriff „Head-mountedDisplay“ (HMD) in Verbindung gebracht (Butterworth et al. 1992). Die technische 
Umsetzung der AR-Technologie ist sowohl bei standardisierten Alltagsbrillen als auch in kompakter Form an einem Helm möglich. Das Display kann in das Sichtfeld des Benutzers ragen oder in durchsichtiger Form die Informationen einblenden (ten Hompel et al. 2011).

Die reale Umwelt wird durch Informationen erweitert, wodurch eine erhöhte Kommissionierleistung im Arbeitsprozess erwartet werden soll. Besonders im Hinblick auf unerfahrene Mitarbeiter oder bei hohen Zeitarbeiterquoten im Unternehmen lässt sich Pick-by-Vision effizient einsetzen. Grund dafür ist u.a. die Prozessführung entlang der Kommissionierung, wodurch eine kognitive Entlastung des Nutzers der Smart Glasses versprochen wird. Solche Anwendungsfälle werden auch als Use Cases bezeichnet und lassen sich als Funktionsfähigkeit einer Smart Glasses in der Pick-by-Vision-Kommissioniermethode widerspiegeln. Mithilfe einer intuitiven Bedienung und dem Einblenden von Informationen in das Blickfeld des Smart-Glasses-Nutzers (Reif 2009) konnten insg. 36 Anwendungsfälle identifiziert werden. Die Anwendungsfälle lassen sich u.a. in Prozessgruppen wie Management, Kommunikation, Value Added Services und phasenspezifische Use Cases einordnen. Darunter fallen Funktionen wie die Mitarbeitersteuerung, Telefonie, Schadensdokumentation und Wegweisung (Niemöller et al. 2017). Die Kommissionierung wird als fertigkeits-, regelbasierte- und informationsintensive Tätigkeit dargestellt, sodass eine technische Unterstützung der Kommissionierung mithilfe von AR und Smart Glasses im Pick-by-Vision-Prozess zu einem der wichtigsten Erfolgsfaktoren gehört (Lolling 2003). Das Einblenden von Informationen im Sichtfeld des Smart Glasses Nutzers ist ausschlaggebend.

\section{Bewertung der Vor- und Nachteile belegloser Kommissioniermethoden}

Um eine Bewertung der Vor- und Nachteile belegloser Kommissioniermethoden durchführen zu können, wird eine Methode zur Wirtschaftlichkeitsbewertung herangezogen. Dabei sollen gesamtorganisatorische Aspekte wie bspw. Lagertypen und IT-Infrastrukturen eines Unternehmens berücksichtigt werden. Das Wirtschaftlichkeitsmodell nach Picot und Reichwald (1984) zielt auf die Wirtschaftlichkeitsbewertung von allgemeinen Unternehmensprozessen ab und betrachtet das Unternehmen aus vier unterschiedlichen Perspektiven. Die Methode soll ein qualitatives Ergebnis erzeugen. Um Erfolgsfaktoren sowie Vor- und Nachteile von definierten Kommissioniermethoden zu bewerten, wurde das Modell im Rahmen dieses Beitrags modifiziert und in Anlehnung an Picot und Reichwald folgende Klassifizierung gewählt:

1. Betrachtung von technikbezogenen Vor- und Nachteilen,

2. Betrachtung von personenbezogenen Vor- und Nachteilen und

3. Betrachtung von finanzbezogenen Vor- und Nachteilen 
Das Modell eignet sich aufgrund seines vordefinierten Betrachtungshorizonts. Dieser verfügt sowohl über monetäre, objektive als auch subjektive Indikatoren zur Wirtschaftlichkeitsbewertung. In der modifizierten Fassung werden personenund finanzbezogene Perspektiven um die technikbezogene Betrachtung ergänzt, da es sich im zu untersuchenden Anwendungsfall um unterschiedliche Technologien in der Kommissionierung handelt. Den Perspektiven des modifizierten Modells werden „Unter-Fragen“ zugeordnet (vgl. Tabelle 1). Diese basieren vornehmlich auf den Erkenntnissen der systematischen Literaturrecherche und ermöglichen die Erstellung eines Fragebogens. Außerdem setzen die Fragestellungen inhaltlich an dem vordefinierten Betrachtungshorizont des angewandten Wirtschaftlichkeitsmodells nach Picot und Reichwald (1984) an.

Tabelle 1. Bewertungsmodell für Kommissioniermethoden, Picot und Reichwald (1984)

\begin{tabular}{|c|c|c|}
\hline $\begin{array}{l}\text { 1. Technikbezogene } \\
\text { Indikatoren }\end{array}$ & $\begin{array}{l}\text { 2. Personenbezogene } \\
\text { Indikatoren }\end{array}$ & $\begin{array}{l}\text { 3. Finanzwirtschaftliche } \\
\text { Indikatoren }\end{array}$ \\
\hline $\begin{array}{l}\text { - Welche Technologie wird ein- } \\
\text { gesetzt? } \\
\text { - Ist die Methode mit einem be- } \\
\text { reits etablierten Barcode- } \\
\text { System kombinierbar? } \\
\text { - Ist die Methode individuell an- } \\
\text { passbar und wird ein ERP- } \\
\text { System benötigt? } \\
\text { - Wie verläuft der Informations- } \\
\text { fluss (Online/Offline)? } \\
\text { - Welche Lagertypen eignen sich } \\
\text { bzw. eignen sich nicht? } \\
\text { - Sind weitere Wearable- } \\
\text { Kombinationen oder Hilfsmittel } \\
\text { möglich/notwendig? }\end{array}$ & $\begin{array}{l}\text { - Wie sieht die allgemeine } \\
\text { Akzeptanz der Mitarbeiter } \\
\text { hinsichtlich der/des Ver- } \\
\text { trauens, Datenschutzes, } \\
\text { Risiken, Chancen gegen- } \\
\text { über der Technologie aus? } \\
\text { - Wie sieht die Ge- } \\
\text { brauchstauglichkeit der } \\
\text { Technologie aus? } \\
\text { - Wie verhält sich der Moti- } \\
\text { vationsfaktor des Perso- } \\
\text { nals? } \\
\text { - Wie gut sind die Anlern- } \\
\text { phasen der Technologie? }\end{array}$ & $\begin{array}{l}\text { - Welche Effizienz- } \\
\text { treiber gibt es? } \\
\text { - Welche Kostentrei- } \\
\text { ber gibt es? } \\
\text { - Wie verhalten sich } \\
\text { durchschnittliche In- } \\
\text { vestitionskosten? } \\
\text { - Wie verhalten sich } \\
\text { laufende Kosten? }\end{array}$ \\
\hline
\end{tabular}

\subsection{Technikbezogene Vor- und Nachteile}

(1) Pick-by-Scan beinhaltet MDE-Geräte in Form von Handhelds als Ausführungstechnologie, die im Laufe eines Kommissioniervorgangs die Auftragsliste ersetzt. Das Display des Gerätes zeigt die zu pickenden Artikel an und wird meistens über physische Tasten bedient. Eine Akkulaufzeit von ca. acht Stunden muss außerdem für einen praxistauglichen Einsatz einer Schicht berücksichtigt werden (Reif 2009). Die Nutzung des MDE-Gerätes, welches ausgereifte Scantechnologie beinhaltet, erfolgt stets mit einer Hand, sodass jeweils die andere Hand nur noch für den Kommissionierprozess genutzt werden kann (Martin 2011). Nachdem die Auftragsstruktur auf den MDE-Geräten eingespielt worden ist, können die Geräte offline verwendet werden. Im Zuge des Scanvorgangs werden die gescannten Daten lokal auf dem Gerät gespeichert und in der Regel nachträglich über eine Docking-Station an den jeweiligen Desktop-PC übertragen. Die MDE-Geräte können 
auch online genutzt werden, um per Funk einen synchronen Datenaustausch zu gewährleisten (ten Hompel et al. 2011). Für den Einsatz von MDE-Geräten sind klassische Boden- und Regallagerungen geeignet. Diesen Lagertyp findet man häufig bei Großhändlern vor. Aufgrund der angebotenen Offline-Lösung der MDE-Geräte, kommen auch Lagervorrichtungen mit niedrigem Digitalisierungsgrad in Frage (Schulte 2009). Die durchgängige Handhabung eines MDE-Gerätes erschwert jedoch Kombinationsmöglichkeiten mit weiteren Wearables. Stattdessen wäre es denkbar, die MDE-Geräte durch alternative Wearables wie bspw. Smart Watches oder Smartphones zu ersetzen (Fraunhofer 2019).

(2) Pick-by-Voice bietet technologisch den größten informativen Unterstützungsgrad (Günthner et al. 2009). Diese Kommissioniermethode beinhaltet ein Headset, ein Mikrofon, ein Sprachterminal und entsprechendes Equipment, um die Ausrüstung am Körper tragen zu können. Dabei kann es sich um einen Tragegurt oder eine Weste handeln (Lydia 2020). Die Technologie umfasst außerdem Automatisierungsmöglichkeiten. Anstelle einer Prozessführung durch einen realen Sprecher, können mithilfe der „Record-and-Play“-Funktion bereits aufgezeichnete Befehle abgerufen werden (Günthner et al. 2009). Die Akkulaufzeiten der SprachTerminals umfassen etwa 14 Stunden (Simões und Späth 2018). Die Pick-byVoice-Kommissioniermethode kann sowohl mit als auch ohne einer Barcodeorientierten Artikelverwaltung integriert werden, da Lagerfach-Prüfziffern per Spracheingabe registriert werden können (Simões und Späth 2018). Der Kommissionierer wird verbal entlang des Prozesses geleitet, während die Aufzeichnung einzelner Prozessschritte der Warenentnahmen in der Lagerverwaltung parallel dokumentiert werden kann. Das Terminal wird per Funk angesteuert und vom Kommissionierer mit einem Taster bedient. Hier besteht die Gefahr, dass „Funklöcher" den Informationsfluss unterbrechen (Arnold et al. 2007). Da sich Headsets und Mikrofonsysteme jedoch in verschiedensten Branchen im Einsatz befinden, kann von einer ausgereiften Technologie ausgegangen werden. Die Anwendung in Lagervorrichtungen mit hohem Geräuschpegel sind für eine akustische Prozessführung ungeeignet. Für Tätigkeitsbereiche, in denen mit Handschuhen gearbeitet werden muss, ist die Bedienung des Terminals ebenfalls unvorteilhaft (Günthner et al. 2009). Außerdem eignet sich die Pick-by-Voice-Kommissioniermethode für Lagertypen mit kurzen Laufwegen. Diese Methode zielt primär darauf ab, die Totzeiten zu reduzieren. Dieser Vorteil kann nicht genutzt werden, wenn die Wegzeiten im Kommissionierungsvorgang überwiegen, wie es bspw. bei offenen Lagertypen oftmals der Fall ist (Simões und Späth 2018). Wearable-Kombinationen oder der Einsatz von Hilfsmitteln wären auch beim Pick-by-Voice aufgrund der freihändigen Vorgehensweise denkbar. Strukturierte Lagerwege mit geeigneten Förderfahrzeugen können lange Wegzeiten reduzieren. Wearable-Kombinationen können die Funktionen einer Quittiertaste ersetzen.

(3) Pick-by-Light strebt eine Prozessführung mit visuellen Markierungen an. Die oftmals in mehreren Farben am Lagerregal befestigten Lämpchen gehören zu einem statisch installierten Pick-by-Light-Kommissioniersystem, welches nur aufwendig umgebaut werden kann. Die klassische Umsetzung eines Pick-by-Light- 
Systems wird durch eine umfangreiche Verkabelung der Regalsysteme ermöglicht. Die voranschreitende Technologie etabliert jedoch bereits Regalbeleuchtung per Funk und ermöglicht eine kabellose Verwendung. Diese ist nur unter erhöhten Installierungskosten umsetzbar (Günthner et al. 2009). Akkulaufzeiten bei funkorientierten Geräten liegen je nach Gebrauch bei sechs Monaten bis zu drei Jahren (L-Mobile 2019). Diese Kommissioniermethode verabschiedet die weit verbreitete Scan-Technologie und ermöglicht eine Kommissionierung ohne Barcodes. An den Regalfächern angebrachte Displays zeigen nach Bestätigung einer Quittierungstaste die zu pickende Anzahl des entsprechenden Artikels an. Die Entnahme des Artikels wird am Regalfach vom Kommissionierer bestätigt (Reif 2009). Dadurch werden die Daten an ein Resource Planning System (ERP)-System gesendet und ermöglichen sowohl einen stationären-/online-basierten Informationsfluss als auch eine synchrone Aufzeichnung von Fehlermeldungen (Günthner 2010). Nach Installation der Regalfächer kann mit erschwerten Anpassungsmöglichkeiten gerechnet werden, da eine nachträgliche Umstrukturierung der Regalsysteme nur aufwendig umsetzbar ist (Reif und Günthner 2009). Dabei kann es sich um die Put-to-Light-Methode handeln. Hierbei wird auf die stationär installierte Technik verzichtet und ein mobiles Kommissionierfahrzeug eingesetzt. Dieses beinhaltet dieselben Komponenten (z.B. Lämpchen) wie ein Pick-by-Light-System (Krajcovic et al. 2014). Die klassische Pick-by-Light-Kommissioniermethode kann sowohl in Kombination mit Lagerregalen im Sinne der „Mann-zu-Ware“-Lösung angewendet werden als auch in Form eines „Kommissioniernests“. Dieses beschreibt eine statische Kommissionierung als „Ware-zu-Mann“-Lösung (Hausladen 2016). Pick-by-Light bietet sich primär bei hohen Umschlagsfrequenzen an. Als Richtwert wird von einer Anzahl von 100 Artikeln pro 30 Meter Regalweg ausgegangen. Ein großes Hindernis ist die gleichzeitige Arbeit von mehreren Kommissionierern im gleichen Regalabschnitt. Erst eine Aufrüstung durch mehrfarbige Lämpchen pro Regalfach würde dies ermöglichen (Günthner et al. 2009). Viele Wearable-Kombinationen in der Kommissionierung streben einen beschleunigten Scan- oder Suchprozess an (Ubimax 2016). Da die Pick-by-Light-Kommissioniermethode keinen Scanprozess beinhaltet, sind verkürzte Laufzeiten mithilfe von Flurfahrzeugen denkbar.

(4) Pick-by-Vision basiert auf dem Einsatz von Smart Glasses. In Form einer Datenbrille werden Auftragsinformationen freigegeben. Die Geräte werden stationär aufgeladen und können eine Akkulaufzeit von zwei bis zwölf Stunden je nach Smart-Glasses-Modell umfassen (Vuzix 2019). Die integrierte Scan-Technologie, die sich in Form einer Kamera oftmals am Brillengestell befindet, deckt den geforderten Bedarf bei der Integration von Digitalisierungsmaßnahmen bspw. im Sinne der Optimierung des Warehouse-Management-Systems ab. Damit ist bspw. die weit verbreitete Nutzung von Barcodes gemeint (Günthner et al. 2009). Das Unternehmen steuert die Auftragsinhalte über ein ERP-System und projiziert die Inhalte auf die Datenbrille. Die Software bzw. das Graphical User Interface lässt sich mit geringem Einsatz von Visualisierungen an die betrieblichen Anforderungen anpassen und benutzerorientiert gestalten (Reif et al. 2009). Für die Informa- 
tionsfreigabe ist ein Online-Empfang im Lagersystem von Nöten, um Picks in Echtzeit zu synchronisieren. Die Smart-Glasses-Integration kann in Verbindung mit der „Online-Ausleuchtung“ somit auch als eine cloud-basierte Lösung interpretiert werden (Ternès und Schieke 2018). Für den Einsatz von Pick-by-Vision bieten sich sowohl statische als auch manuelle Materialflusstypen an. Vollautomatische „Ware-zum-Mann“-Lösungen verhindern jedoch Einsparungen von Wegzeiten, die durch den Einsatz von Smart Glasses ermöglicht werden. Die Wegzeiten gehören in den meisten Lagersystemen zu den zeitintensivsten Aufwendungen (Arnold et al. 2007). Somit würde das Optimierungspotenzial der Kommissionierleistung von Smart Glasses nicht genutzt werden. Geeignete Lagertypen hingegen kennzeichnen sich durch klassische „Mann-zu-Ware“-Lösungen, wie das Fachbodenregallager und das Durchlaufregallager. Weiterhin bieten sich Einsätze sowohl in einstufigen-, als auch in mehrstufigen Kommissioniersystemen an (Reif 2009). Smart Glasses ermöglichen somit mithilfe von Wearable-Kombinationen eine Nutzung von neuen Potenzialen zur Effizienzsteigerungen. Hierfür werden bspw. Smart Watches oder Ring-Scanner verwendet, um schnellere Scanvorgänge ermöglichen zu können (Dataphone 2017). Um bspw. die physische Interaktion mit der Smart Glasses zu ersetzen, können zudem auch Smart Watches in Betracht gezogen werden. Dabei könnte sich die Pick-by-Vision-Kommissioniermethode an der „Non-speech text entry method“ vom KAIST HCI Lab bedienen. Diese stellt die Interaktionsmöglichkeiten mit Smart Watches dar. Die Eingaben in Form von Tippen oder Streichen auf der Smart Watch werden in Echtzeit auf das Display der Smart Glasses projiziert, sodass diese nicht weiter physisch bedient werden muss (Ahn et al. 2017). Andernfalls können moderne Hilfsmittel, wie technologisch fortgeschrittene Gabelstapler, heutzutage Technologien wie automatische Wegfindung oder Kamerasysteme beinhalten. Dadurch werden Use-Cases von Smart Glasses ersetzt (Günthner et al. 2009).

\subsection{Personenbezogene Vor- und Nachteile}

(1) Pick-by-Scan beinhaltet tragbare Computer und ermöglicht individuelle Anpassungsmöglichkeiten. MDE-Geräte können auch mit Kamera- und Mikrofonsystemen ausgestattet sein. Dies gilt vor allem für Pick-by-Scan-Systeme dessen ausführende Scantechnologie durch Smartphones o.Ä. ersetzt wurde (Liening 2018). Die Gebrauchstauglichkeit fällt bei der ausführenden Technologie im Pick-byScan relativ gering aus. Aufgrund der notwendigen Handhabung eines MDE-Gerätes ist stets mindestens eine Hand des Kommissionierers besetzt. Dadurch wird der Arbeitsprozess punktuell unterbrochen. Ferner muss sich der Kommissionierer mit dem Gerät vor produktiver Nutzung auseinandersetzen und ist nicht frei von sprachlichen Barrieren. Dies kann z.B. die Nutzung von Abkürzungen betreffen (Reif 2009). Durch die meist schwarze Schrift auf grauem Hintergrund, kann es bei hell ausgeleuchteten Lagerhallen oftmals zu sog. Reflexblendungen kommen (Günthner et al. 2009). Sobald ein MDE-Gerät durch ein alternatives Wearable, wie bspw. ein Smartphone oder eine Smart Watch, ersetzt wird, kann dies zu wei- 
teren Vorteilen führen. Der Kommissionierer ist i. d. R. mit solchen Technologien vertraut, da diese auch im privaten Umfeld genutzt werden (Liening 2018). Dadurch wären ein beschleunigter Anlernprozess und ein erhöhter Motivationsfaktor denkbar.

(2) Pick-by-Light-Systeme beinhalten festinstallierte Quittiertasten am Regalfach, sodass Personal- und Störstatistiken abgerufen werden können (Martin 2011). Dadurch ist eine ständige Überwachung des Arbeitsprozesses gewährleistet. Mithilfe des etablierten Fragebogens hinsichtlich der Nutzer-Akzeptanz, wurde bereits Skepsis gegenüber solchen Technologien mit „Überwachungscharakter“ erkannt. Die Überwachungsproblematik kann auch beim Einsatz von Pick-byLight-Systemen vermutet werden. Pick-by-Light beruht auf dem optischen Sinn des Menschen, mit dem er am besten seine Umgebung wahrnimmt. Dadurch wird die Einsatzfähigkeit des Mitarbeiters trotz hohen Aufwands gewährleistet. Jedoch kommt es oftmals durch eine falsche Bedienreihenfolge zu Zählfehlern (Reif 2009). Diese Kommissioniermethode baut ebenfalls auf einer visuellen Prozessführung auf, wodurch schnellere Anlernzeiten ermöglicht werden und Fehlpicks gegenüber konventionellen Kommissioniermethoden wie dem Pick-by-Paper- oder Pick-by-Scan-Ansatz grundsätzlich reduziert werden sollen (Krajcovic et al. 2014). Dadurch, dass der optische Suchaufwand erhöht wird, ist mit Konzentrationseinschränkungen und erhöhter Ermüdung der Mitarbeiter im Laufe eines Arbeitstages zu rechnen (Günthner et al. 2009).

(3) Pick-by-Voice verabschiedet sich gänzlich vom optischen Sinn des Menschen und baut auf einer akustischen Prozessführung auf. In der Regel basiert sie auf kurzen und präzisen Anweisungen, was jedoch zu einer Abschottung der Kommunikation zwischen den Mitarbeitern führen kann. Mitarbeiter mit Migrationshintergründen, die eine undeutliche Sprachweise oder ein mangelndes Verständnis der angewandten Sprache aufweisen, könnten sich mit dieser Technologie ebenfalls unwohl fühlen. Andernfalls sind Sprachanweisungen in unterschiedlichen Sprachen möglich und eine innovative Spracherkennungstechnologie beugt dieser Problematik vor (Lydia 2020). Ist eine störfreie Kommunikation gegeben, sind sowohl Hände als auch Augen frei, wodurch sich der Kommissionierer gänzlich auf den Arbeitsprozess konzentrieren kann. Die Gebrauchstauglichkeit ist sehr hoch, sodass gewisse Bewegungs- und Informationsabläufe parallel möglich sind (Reif 2009). Einerseits weist diese Kommissioniermethode aufgrund genauer akustischer Anweisungen geringe Anforderungen an den Nutzer auf, sodass eine beschleunigte Anlernphase auch hier üblich ist. Andererseits ist oftmals jedoch ein Sprachtraining notwendig, welches eine zügige Integration der Technologie erschwert (Reif 2009). Die Mitarbeiter müssen sich bei automatisch abgespielten Texten von monotonen Stimmen leiten lassen, was zu einer Demotivation führen kann (Günthner et al. 2009). Der hohe Unterstützungsgrad wird jedoch oftmals durch aufwendiges Equipment dargestellt werden. Das Headset, Gurt/Weste, Terminal und ggfs. ein Scanner könnte für Mitarbeiter zu überladen wirken.

(4) Pick-by-Vision ist hinsichtlich des Datenschutzes des Personals aufgrund der Kamera- und Mikrofronausstattung eine sehr umstrittene Kommissionierme- 
thode. Daher wurden Labortests auf Basis eines 8-Punkte-Prüfplans, untermauert durch einen etablierten Fragebogen bzgl. der Akzeptanz von Mitarbeitern, im Rahmen des GLASSHOUSE-Projekts angewendet. Der Labortest beinhaltet die Montage einer hölzernen Lokomotive. Ein Proband wird mit der Vuzix M100 Smart Glasses ausgestattet, die ihn bei der Montage unterstützt. Bei dieser Methode handelt es sich um die aus acht etablierten Akzeptanzmodellen hergeleitete Akzeptanztheorie von Venkatesh et al. (2003), die u.a. den erwarteten Aufwand und den sozialen Einfluss testet. Um den Eingriff von Technologien in die Privatsphäre des Nutzers zu integrieren, wird das Modell von Zhou (2012) erweitert (Venkatesh et al. 2003). Damit werden die zu untersuchenden Faktoren um das Vertrauen und das wahrgenommene Risiko ergänzt. Einen weiteren Faktor für die Implementierung einer neuen Kommissioniermethode stellt deren Gebrauchstauglichkeit dar (Niemöller et al. 2018). Studenten der Wirtschaftswissenschaften als Probanden der Untersuchung nehmen tendenziell weniger Risiken wahr und vertrauen den Smart Glasses. Dabei bezieht sich die Untersuchung auf den Schutz persönlicher Nutzerdaten. Dennoch vermutet die Gruppe der Probanden, dass persönliche Daten über Standortdienste und Tonaufnahmen der Smart Glasses kontrolliert und dokumentiert werden können (Berkemeier et al. 2017). Der erwartete geringere Aufwand und die leichte Erlernbarkeit aufgrund einer digitalen Prozessführung der Pick-by-Vision-Technologie ermöglicht ebenfalls einen wahrgenommenen Nutzen bei den Probanden. Beobachtungen und Aussagen zufolge mussten sich einige Probanden jedoch nach Nutzung der Smart Glasses die Augen reiben. Gelegentlich werden die Augen geschlossen, das Gerät muss nachgerückt, und zwischenzeitlich müssen die Smart Glasses sogar abgesetzt werden. Um konkrete Stärken in der Gebrauchstauglichkeit zu identifizieren, müssen die Smart Glasses einer Langzeitbeobachtung unterzogen werden (Berkemeier et al. 2017). Eine Steigerung der Motivation von Smart-Glasses-Nutzern konnte bereits in weiteren Untersuchungen festgestellt werden. Dabei konnte eine Steigerung von etwa $20 \%$ verifiziert werden (Cinovation 2018). Grund dafür könnte einerseits die persönliche Affinität zur innovativen Technologie sein. Andererseits basiert Pick-byVision u.a. auch auf der Nutzung von Gamification, wodurch Anwender durch spielerische Symbole dazu motiviert werden, effizienter zu arbeiten (Wood und Reiners 2012).

\subsection{Finanzwirtschaftliche Vor- und Nachteile}

Im Zuge einer systematischen Literaturrecherche haben sich einige Effizienztreiber ergeben, die die Kommissionierleistung primär beeinflussen. Diese können sich bspw. auf den Return on Investment (ROI) der unterschiedlichen Kommissioniertechnologien auswirken. Dabei handelt es sich primär um die erhöhte Arbeitsgeschwindigkeit, die eine erhöhte Produktivität bzw. eine Verringerung des Arbeitsaufwands mit sich bringt (Tompkins et al. 2003). Als weiterer Effizienztreiber ergibt sich die Fehlerquotenreduzierung, die Referenzwerten aus der Praxis 
zu Folge grundsätzlich eine höhere Rendite bzw. den sog. „Return“ erwarten lässt (Cinovation 2018).

(1) Bei der Pick-by-Scan-Kommissioniermethode werden primär die Hardwarekosten für die MDE-Geräte betrachtet. Da grundsätzlich mit der Schnittstelle zu einem ERP- bzw. Lagerverwaltungssystem in beleglosen Kommissioniersystemen zu rechnen ist, wird dieser Aspekt in der Kostenstruktur im Weiteren nicht berücksichtigt. Jedoch beinhaltet die Pick-by-Scan-Kommissioniermethode die Möglichkeit, offline zu kommissionieren, wodurch IT-Kosten eingespart werden können (Arnold et al. 2007).

(2) Pick-by-Voice beinhaltet einen erhöhten informatorischen Unterstützungsgrad, welcher durch ein umfangreiches Equipment gewährleistet wird. Dadurch können die Kosten für die Hardware relativ hoch ausfallen. Dem wirkt jedoch eine verkürzte Anlernphase entgegen, welche den Effizienztreibern zugeordnet werden kann. Hier können geübte Sprecher neue Mitarbeiter durch den Prozess führen und zeitlichen Aufwand einsparen. Handelt es sich jedoch um unausgereifte Technologie oder eine hohe Anzahl an „Funklöcher“, so kann sich der zeitliche Aufwand durch wiederholende Befehle erhöhen. Pick-by-Voice weist in der Anwendung den besten Kosten-/Nutzenfaktor auf (Günthner et al. 2009).

(3) Bei Pick-by-Light-Systemen können die laufenden Kosten den einzelnen Lagerfächern zugeordnet werden. Dabei handelt es sich um Kosten pro Fach, die bei wachsendem Sortiment progressiv ansteigen. Außerdem fallen durch die Sicherstellung einer langfristigen Funktionsfähigkeit der Systeme relativ hohe Wartungskosten an (Reif and Günthner 2009). Die umfangreichsten Kosten sind im Pick-by-Light-System i. d. R. jedoch die Installationskosten (Günthner et al. 2009).

Kurze Anlernzeiten führen auch hier zu einem Einsparungspotenzial. Bei erhöhten Personalwechseln, wie bspw. beim Einsatz von Zeitarbeitern, wirkt sich dieser Aspekt auch langfristig positiv auf den zu erwartenden Return aus. Andererseits erhöht sich der optische Suchaufwand durch mehrere aufleuchtenden Lämpchen im selben Regalgang, wodurch zusätzlicher Zeitaufwand zu erwarten ist (Günthner et al. 2009). In Tabelle 2 werden die Kostensätze der einzelnen Kommissioniermethoden nach Reif (2009) zusammengefasst.

Tabelle 2. Kostensätze belegloser Kommissioniermethoden nach Reif (2009)

\begin{tabular}{lrcr}
\hline Methode & \multicolumn{1}{c}{ Pick-by-Scan } & Pick-by-Light & \multicolumn{1}{c}{ Pick-by-Voice } \\
\hline Fixkosten & $10.000-20.000 €$ & $10.000-20.000 €$ & $10.000-20.000 €$ \\
Kosten pro Benutzer & $5.000-6.000 €$ & $100 €($ pro Fach) & $3.000-10.000 €$ \\
\hline
\end{tabular}

In Anlehnung an eine Kosteneinschätzung von Reif im Jahre 2009 wird im weiteren Verlauf des Abschnitts ein Kostenmodell abgeleitet (vgl. Abb. 4). In der Regel unterscheiden sich die Preise von Anbieter zu Anbieter. Daher bietet es sich an, einen Angebotsvergleich mehrerer Anbieter durchzuführen.

(4) Um eine Kostenstruktur für die Pick-by-Vision-Kommissioniermethode zu ermitteln, wurde ein Angebotsvergleich mehrerer Anbieter durchgeführt. Die Ge- 
samtkosten können sich aus einmaligen und laufenden Kosten zusammensetzen. Unter einmaligen Kosten fallen Integrations-, Hard- und Softwarekosten. Lizenzkosten können sowohl einmalig in Pauschalen als auch laufend pro Benutzer abgerechnet werden. $\mathrm{Zu}$ den laufenden Kosten zählen außerdem sonstige Ausgaben (z.B. für Wartung und Instandhaltung). Auch einem Pick-by-Vision-Kommissioniersystem sind sonstige Effizienztreiber wie eine kurze Anlernphase zuzuordnen. Mithilfe des Einsatzes von spielerischen Symbolen und Inhalten wird außerdem mit einem Motivationszuwachs der Mitarbeiter gerechnet. Diese Methode ist unter dem Begriff „Gamification“ bekannt (Wood und Reiners 2012). In Tabelle 3 sind beispielhaft die Fehlerquoten der beleglosen Kommissioniermethoden aufgeführt, welche sich besonders auf einen erwarteten ROI auswirken können. Stichprobenartig wurden jeweils drei Literaturquellen herangezogen.

Tabelle 3. Fehlerquoten belegloser Kommissioniermethoden, stichpunktartiger Vergleich

\begin{tabular}{|c|c|c|c|}
\hline Methode & $\begin{array}{l}\text { Durchschnittlich } \\
\text { reduzierte Fehlerquote }\end{array}$ & Quellen & Fehlerquoten \\
\hline \multirow{3}{*}{ Pick-by-Scan } & \multirow{3}{*}{$0,393 \%$} & Lolling 2003 & 0,36 \\
\hline & & ten Hompel, M., Schmidt 2004 & 0,46 \\
\hline & & Günthner et al. 2009 & 0,36 \\
\hline \multirow{3}{*}{ Pick-by-Voice } & \multirow{3}{*}{$0,1433 \%$} & Lolling 2003 & 0,10 \\
\hline & & Reif 2009 & 0,25 \\
\hline & & ten Hompel, M., Schmidt 2004 & 0,08 \\
\hline \multirow{3}{*}{ Pick-by-Light } & \multirow{3}{*}{$0,326 \%$} & Lolling 2003 & 0,40 \\
\hline & & Reif 2009 & 0,25 \\
\hline & & ten Hompel, M., Schmidt 2004 & 0,08 \\
\hline \multirow{3}{*}{ Pick-by-Vision } & \multirow{3}{*}{$0,1836 \%$} & Guo et al. 2014 & 0,0075 \\
\hline & & Göpfert and Kersting 2017 & 0,125 \\
\hline & & Günthner et al. 2009 & 0,12 \\
\hline
\end{tabular}

Hinzuzufügen ist, dass es eine „30 \% -Regel“ in Unternehmen gibt, die besagt, dass sich durch papierlose Lagerprozesse die Produktivität um $30 \%$ erhöht und die Fehlerrate um 30 \% reduziert (Reif 2009). Als Implikation für die Entscheider in der Praxis lassen sich somit einige Handlungshinweise ableiten. Durch Integration einer Vielzahl an Einflussfaktoren und Vor- und Nachteilen der einzelnen Kommissioniermethoden, wird ein Einordnungsrahmen für Unternehmen in der Praxis erstellt. Unabhängig von der gewählten Kommissioniermethode fallen entsprechende Anschaffungs- und laufende Kosten an. Unter Berücksichtigung des Kommissionierungsbedarfs, des Lagertyps und der Unternehmensstruktur können sich die Kosten unterschiedlich entwickeln. In Anlehnung an die Kostenaufstellung nach Reif wird unter Berücksichtigung der Eigenschaften der Kommissioniermethoden folgendes Kostenmodell abgeleitet (vgl. Abb. 4). 


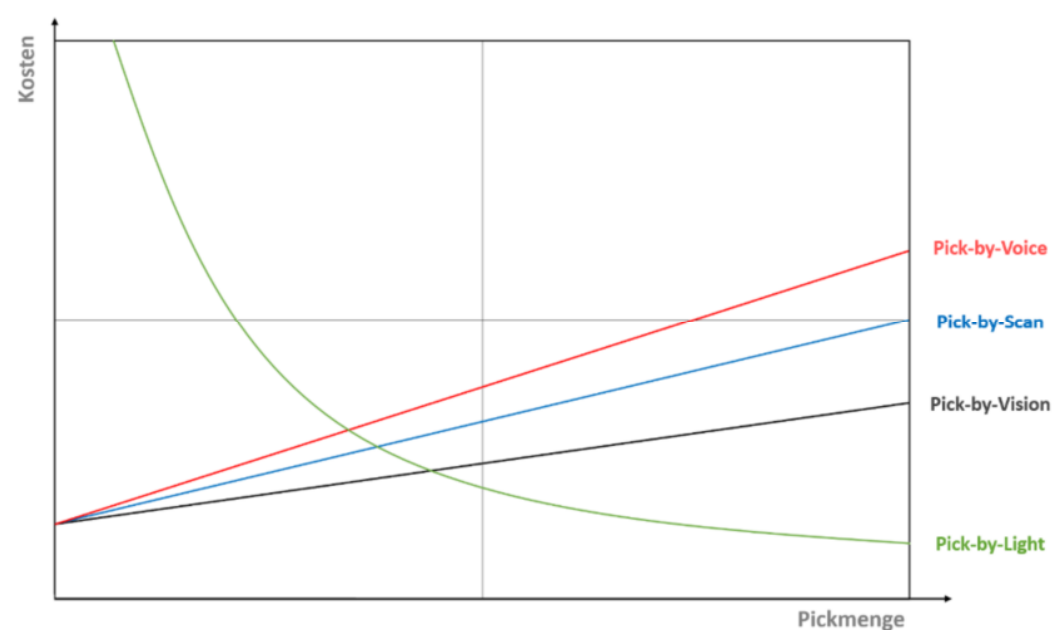

Abb. 4. Kommissioniermethoden - Kostenmodell

\subsection{Implikationen für die Forschung}

Grundsätzlich lässt sich in nahezu allen innovativen Technologien Forschungspotenzial erkennen. Der Informationsgrundlage zur Folge, ist es das Hardware-Design bzw. die Gebrauchstauglichkeit der Pick-by-Vision-Technologie, die ausbaufähig ist. Aus rechnerischer Sicht und unter Berücksichtigung von Referenzwerten aus der Praxis überwiegen die Vorteile des Einsatzes von Smart Glasses oftmals. Diese Vorteile sind für ein Unternehmen jedoch nicht greifbar, wenn die Mitarbeiter als ausführende Arbeitskräfte keinen störfreien Einsatz der Smart Glasses gewährleisten können. Sowohl die Ergonomie als auch eine ausgereifte Hardware sind Voraussetzung für eine vorteilhafte Integration von Smart Glasses in einem Unternehmen.

Um konkrete ergonomische Schwachstellen zu identifizieren, sind weitere Tests hinsichtlich der Gebrauchstauglichkeit durchzuführen, da es in der Praxis an Langzeitbeobachtungen mangelt. Aus den Kommentaren des Labortests, wie bspw. Nachrücken, Augen reiben und Augen zudrücken, ist deutlich zu erkennen, dass es Verbesserungen hinsichtlich des Displays erfordert. Im Zuge der Forschung bzgl. Anpassungsmöglichkeiten des Displays, müssen jedoch Richtlinien berücksichtigt werden. Diese Richtlinien können sowohl auf herstellerbasierten Grundlagen aufbauen als auch unter Berücksichtigung der Sehfähigkeit des menschlichen Auges festgelegt worden sein. Dabei wird bspw. ein Mindestabstand vom Auge zu einer Brille mit $12 \mathrm{~mm}$ festgelegt (Aukstakalnis 2016).

Einige Probanden haben die Smart Glasses im Arbeitsprozess versehentlich ausgeschaltet oder nach Interaktionsmöglichkeiten gesucht. Um solche Fehler zu beheben, können Wearable-Kombinationen als sinnvoll erachtet werden. Hierbei stellt sich die Frage, wie effizient solche Wearable-Kombinationen unter Berücksichtigung des gesamten Kommissionierungsprozesses eingesetzt werden können. 
Um die physische Interaktion mit den Smart Glasses ersetzen zu können, können bspw. Smart-Watches-Ring-Scanner in Betracht gezogen werden.

Ein Entwicklungsrahmen bzw. Framework zur strukturierten Entwicklung von Applikationen an Smart Glasses ist notwendig. Dabei müssen standardisierte ITSchnittstellen berücksichtigt werden. Es ist davon auszugehen, dass ab bestimmten Kapazitätsgrenzen, wie bspw. im Bereich des Wareneinsatzes oder der personellen Ressourcen, umfangreiche Logistiksysteme notwendig sind. Diese sind nur handhabbar, wenn die Lagersysteme einen Online-Empfang ermöglichen, um eine Datensynchronisierung in Echtzeit zu gewährleisten. Hiermit werden sowohl die Voraussetzungen für eine ununterbrochene Wertschöpfungskette als auch für den Einsatz von Smart Glasses geschaffen. Obwohl der Forschungsrahmen auf logistische Anwendungsbereiche reduziert wurde, ist davon auszugehen, dass der effiziente Einsatz der Smart Glasses entlang der gesamten Wertschöpfungskette an weiterer Forschung bedarf. Die Anwendungsbereiche etablierten sich oftmals nur durch Experimente. Langzeitbeobachtungen und Praxistests können neue Anwendungsfelder ermitteln oder bereits vorhandene Use Cases adaptieren.

\section{Zusammenfassung und Diskussion}

Nach Anwendung des modifizierten Bewertungsmodells in Anlehnung an Picot und Reichwald (1984) konnten Implikationen für Theorie und Praxis formuliert werden. Zum einen entsteht ein theoretischer Beitrag für die Forschung in Form einer (1) strukturierten Darstellung des Smart-Glasses-basierten Pick-by-VisionPotenzials gegenüber am Markt etablierten Technologien. Dabei werden Erfolgsfaktoren klassifiziert und aus drei betriebswirtschaftlich relevanten Perspektiven betrachtet. Im Zuge ansteigender Digitalisierungsmaßnahmen in kleinen und großen Unternehmen, fokussiert sich dieser Beitrag auf die standardisierten beleglosen Kommissioniermethoden nach Günthner. Die Kommissionierung gehört zu einem der ressourcenintensivsten Prozesse in der Logistik, wodurch (2) ein Einordnungsrahmen mit wettbewerbsrelevanten Handlungshinweisen entlang des angewendeten Bewertungsmodells begründet ist.

Die Relevanz der Arbeit zeichnet sich außerdem durch die umfassende Datengrundlage aus. Während mithilfe einer systematischen Literaturrecherche gängige Definitionen und Kriterien erhoben worden sind, decken Labortests, etablierte Fragebögen und die Anbieterrecherche praxisorientierten Ergebnisse ab.

Die Intention dieses Beitrags beruht auf dem Megatrend „Digitalisierung“ und beleuchtet im Zeitalter der Industrie 4.0 die Optimierungspotenziale betriebswirtschaftlich relevanter Arbeitsprozesse durch den Einsatz von Smart Glasses.

Kritisch zu betrachten ist die Aussagekraft der Vor- und Nachteile der Pick-byVision-Kommissioniermethode, da nur wenige Langzeitbeobachtungen existieren. Oftmals sind es nur experimentelle Untersuchungen hinsichtlich einzelner Aspekte, welche die Potenziale der Smart Glasses in der Logistik begründen. Die im Gegensatz dazu standardisierten Kommissioniertechnologien am Markt stellen 
somit die Vergleichbarkeit der Ergebnisse in Frage. Weiterhin sind die Auswirkungen auf die Geschäftsprozesse unklar, die durch den Einsatz von Smart Glasses in der Kommissionierung hervorgerufen werden können. Anwendungsfälle wie die automatische Kontroll- und Dokumentationsfunktion der Smart Glasses, wirken sich entsprechend auf die IT-Struktur aus, wodurch gegebenenfalls aufwendigere Prozesse angestoßen werden können. Es entsteht jedoch nicht nur aus technischer Sicht Diskussionsbedarf. Die datenschutzrechtlichen Anforderungen an Smart Glasses sind, wie die Umfrage ergeben hat, sehr umstritten und werfen die Frage auf „Wie kann ein datenschutzkonformes System gestaltet werden?“ bzw. „Wie kann das Vertrauen der Smart-Glasses-Nutzer gewonnen werden?“ Ob kleine „Mute-Symbole“, die visualisieren, dass eine Sprach- oder Videoaufnahme deaktiviert ist, ausreichen, kann nicht festgelegt werden. Untersuchungsbedarf ist bzgl. des „Überwachungscharakters“ bekannter Maßnahmen zur Gewährleistung des Datenschutzes im Bereich der Smart-Glasses-Anwendung erforderlich.

Abschließend kann im Rahmen der finanzbezogenen Perspektive sowohl mit Effizienz- als auch mit Kostentreibern eine Wirtschaftlichkeitsbewertung durchgeführt werden. Diese basiert meist auf experimentellen Ergebnissen oder auf Referenzwerten aus der Praxis. Weiterhin wurden sonstige Einflussgrößen, wie der Motivationsfaktor der Mitarbeiter oder die Anlernphasen, erwähnt. Dies impliziert für die weitere Forschung, dass ggfs. weitere Einflussgrößen bzw. Effizienztreiber zu ermitteln sind. Schließlich konnte ein Kostenmodell aufgrund eines Angebotsvergleichs und unter Berücksichtigung des Bewertungsmodells abgeleitet werden. Die dahinterstehende Kostenstruktur kann als Basis für eine Wirtschaftlichkeitsbewertung weitere Anwendung finden.

\section{Ausblick}

Es kann davon ausgegangen werden, dass das Potenzial von Smart-Glasses-basierten Kommissioniermethoden tendenziell steigt. Im Zeitalter der Industrie 4.0 ist ein stetig voranschreitender Forschungsgrad in Bezug auf innovativen Technologien zu beobachten. Die Verknüpfung mit allgemeinen Digitalisierungsmaßnahmen und dem Einsatz von intelligenten Technologien entlang einer Wertschöpfungskette können vielseitige Anwendungsfelder etablieren. Dies gilt jedoch nicht nur für den Einsatz von Smart Glasses. Neben den bereits vorgestellten Kommissioniermethoden werden neue innovative Techniken entwickelt und erprobt. Ein weiterer neuartiger Ansatz für informationstechnische Kommissioniermethoden umfasst bspw. die Pick-by-Watch-Kommissioniermethode. Hierbei werden dem Kommissionierer die benötigten Informationen über eine Smart Watch angezeigt. Interaktionen erfolgen über die Touch-Funktion. Dadurch ist eine Synchronisierung der Bestände in Echtzeit durch ein Warenwirtschaftssystem möglich (Bächler 2017). Denkbar ist hierbei eine überdurchschnittlich positive Akzeptanz seitens der Nutzer, da die Smart Watch auch außerhalb beruflicher Nutzung als etabliertes Wearable eingestuft werden kann (Ahn et al. 2017). 
Die Pick-by-Beamer-Kommissioniermethode beschäftigt sich, ähnlich wie beim Pick-by-Light, mit direkt am Regal visualisierten Anweisungen. Mit Signalleuchten wird ein Lichtkegel auf ein entsprechendes Regal projiziert. Mithilfe eines auf einem Kommissionierwagen befestigten Bildschirms werden sämtliche Auftragsinformationen freigegeben. Die Innovation hierbei liegt im rotierenden Laserscanner, der am Beamer befestigt ist und manuelle Zugriffe eines Kommissionierers erfassen kann (Bächler 2017).

Das Pick-by-Point-Prinzip basiert auf einer beweglichen Lichtquelle, die ein Entnahmefach markiert und von einem Kommissionierer, ausgestattet mit drahtlosen Kopfhörern, aufgesucht wird. Flexibilität steht auch hier im Vordergrund.

Somit gelten bereits vorhandene Technologien als Grundlage und motivieren die Wissenschaft dazu, weitere Effizienztreiber für den Einsatz von modernen Kommissioniermethoden zu erforschen. Durch Betrachtung der Pick-by-VisionTechnologie kann mithilfe von Best Practices aus Theorie und Praxis ebenfalls weitere Implikationen für die Forschung abgeleitet werden. Dies begründet ein Zusammenspiel aus glaubhafter und vergleichbarer Datengrundlage. Die Grundlage baut auf theoretische Inhalte aus Fachliteratur, Referenzwerten und bereits etablierten Modellen aus der Praxis auf. Die Anwendung von Bewertungsmodellen auf Basis einer Wirtschaftlichkeitsbewertung mit relativ betrachteten Potenzialen und künftige Langzeitbeobachtungen führen zu praxisorientierten Best Practices. Ergebnisse auf Basis von Labortests sind der Auswertung lediglich zu ergänzen, und sollten nicht als primäre Datengrundlage dienen. Die Ableitung eines Frameworks würde somit Vorteile für künftige Forschungsarbeiten in diesem Bereich vereinfachen. Das Ergebnis könnte als (a) Referenzmodell für die Bewertung von Technologien in der Logistik umgesetzt werden. Weiterhin ist (b) ein übergreifendes Modell auf den Einsatz von Technologien im produzierenden Gewerbe denkbar. Logistiktrends und die voranschreitende Technologie decken Forschungsbereiche der Wirtschaftsinformatik auf, die bei der Gestaltung des Einsatzes von Smart Glasses einbezogen werden sollten. Die Forschungsbereiche beinhalten ebenfalls das Dienstleistungsmanagement, die Informationsprivatheit sowie Aufgaben digitaler Arbeit und fördern künftig die Umsetzung von Digitalisierungsmaßnahmen in der Praxis.

\section{Literatur}

Ahn S, Heo S, Lee G (2017) Typing on a Smartwatch for Smart Glasses. In: Subramanian S, Steimle J (Eds) Proceedings of the 2017 ACM International Conference on Interactive Surfaces Spaces. ACM, Brighton. doi:10.1145/3132272.3134136

Almeida DLM de, Ferreira JCE (2009) Analysis of the Methods Time Measurement (MTM) Methodology through its Application in Manufacturing Companies. In: Flex Automation Intelligent Manufactoring. FAIM, United Kingdom, Middlesbrough Arnold D, Isermann H, Kuhn A, Tempelmeier H, Furmans K $(2007,2008)$ Handbuch Logistik. Springer, Berlin Heidelberg 
Aukstakalnis S (2016) Practical Augmented Reality: A Guide to the Technologies, Applications, and Human Factors for AR and VR (Usability). Addison Wesley, Boston

Bächler AM (2017) Entwicklung und Evaluierung eines nutzerzentrierten Assistenzsystems zur Unterstützung von leistungsgeminderten Mitarbeitern bei manuellen Kommissioniertätigkeiten. Dissertation, Technische Universität Ilmenau

Berkemeier L, Werning S, Zobel B, Ickerott I, Thomas O (2017) Der Kunde als Dienstleister: Akzeptanz und Gebrauchstauglichkeit von Smart Glasses im Self-Service. HMD Praxis der Wirtschaftsinformatik 54:781-794

Bode W, Preuß R (2004) Praxishandbuch Intralogistik. Wirtschaftsverlag W.V., Suhl

Butterworth J, Davidson A, Hench S, Olano MT (1992) 3DM: a three dimensional modeler using a head-mounted display. Proc 1992 Symp Interact 3D Graph - SI3D '92 135138. doi:10.1145/147156.147182

Cinovation (2018) Make by Vision, https://www.cinovation.de/web/index.php/technics/make-by-vision, abgerufen am 10.12.2019

Dataphone (2017) Neue Innovationsfelder durch Smart Glasses und Customer Devices, https://lineapro.net/media/files/DIWA_SmartGlasses_GS1ForumIntralogistics2017.pdf , abgerufen am 07.07.2020

Dekker R, Koster MBM de, Roodbergen KJ, Kalleveen H van (2004) Improving OrderPicking Response Time at Ankor's Warehouse. INFORMS Journal on Applied Analytics 34:245-329. doi:10.1287/inte.1040.0083

Ehrmann H (2017) Logistik. NWB, Herne, 9. Auflage

Elbestawi M, Centea D, Singh I, Wanyama T (2018) SEPT Learning Factory for Industry 4.0 Education and Applied Research. Procedia Manufacturing 23:249-254. doi:10.1016/j.promfg.2018.04.025

Gleißner H, Femerling C (2008) Logistik. Gabler, Wiesbaden

Göpfert I, Kersting R (2017) Organisation und Forschungsdesigns der Zukunftsforschung. In: Göpfert I, Kersting R (Hrsg) Wie Unternehmen in die Zukunft blicken. Springer, Wiesbaden, 7-27

Gudehus T (2010) Logistik: Grundlagen - Strategien - Anwendungen, 4. Ausgabe. Springer, Berlin Heidelberg

Günthner WA, Blomeyer N, Reif R, Schedlbauer M (2009) Pick-by-Vision: Augmented Reality unterstützte Kommissionierung. In: Günthner WA (Hrsg) fml - Lehrstuhl für Fördertechnik Materialfluss Logistik. Technische Universität München

Guo A, Starner T, Raghu S, et al (2014) A comparison of order picking assisted by head-up display (HUD), cart-mounted display (CMD), light, and paper pick list. Proc 2014 ACM Int Symp Wearable Comput - ISWC '14 71-78

Hausladen I (2016) IT-gestützte Logistik. Gabler Verlag, Wiesbaden

Klötzer C, Pflaum A (2017) Toward the Development of a Maturity Model for Digitalization within the Manufacturing Industrys Supply Chain. In: Bui TX, Sprague R (Eds) Proceedings of the 50th Annual Hawaii International Conference on System Sciences. University of Hawaii, Manoa

Krajcovic M, Gabajova G, Micieta B (2014) Order Picking using Augmented Reality. Communications - Scientific Letters of the University of Zilina 16(3A):106-111

L-Mobile (2019) L-Mobile Pick-by-Light. https://www.l-mobile.com/geschaeftsfelder/ digitalisierte-produktion/funktionen/materialfluss/pick-by-light/\#infothek, abgerufen am 10.12.2019 
Liening B (2018) MDE Geräte oder Smartphones? https://www.storesshops.de/technology/mde-geraete-oder-smartphones/, abgerufen am 10.12.2019

Lolling A (2003) Analyse der menschlichen Zuverlässigkeit bei Kommissioniertätigkeiten. Shaker Verlag, Dortmund

Lydia (2020) Voxter Elite. https://www.lydia-voice.com/de/voice-hardware/voxter-elite/, abgerufen am 07.07.2020

Martin H (2011) Kommissioniersysteme. In: Martin H (Hrsg) Transport- und Lagerlogistik. Vieweg+Teubner Verlag, Wiesbaden, 8. Auflage

Martin H (2014) Kommissioniersysteme. In: Martin H (Hrsg) Transport- und Lagerlogistik. Vieweg+Teubner Verlag, Wiesbaden, 9. Auflage

Niemöller C, Schomaker T, Thomas O (2018) Einsatz von Smart Glasses in Unternehmen. Analyse und Gestaltung von Geschäftsmodellen. In: Thomas O et al. (Hrsg) Digitalisierung in der Aus- und Weiterbildung. Springer, Berlin, 170-181

Niemöller C, Zobel B, Berkemeier L, Metzger D, Werning S, Adelmeyer T, Ickerott I, Thomas O (2017) Sind Smart Glasses die Zukunft der Digitalisierung von Arbeitsprozessen? Explorative Fallstudien zukünftiger Einsatzszenarien in der Logistik. In: Leimeister J, Marco BW (Hrsg) 13. International Conference on Wirtschaftsinformatik (WI). St. Gallen, 410-424

Picot A, Reichwald R (1984) Bürokommunikation. Leitsätze für den Anwender. CWPublikationen Verlagsgesellschaft GmbH, München

Pfohl H (2018) Logistiksysteme. Springer, Heidelberg

Reif R (2009) Entwicklung und Evaluierung eines Augmented Reality unterstützten Kommissioniersystems. Dissertation, Technische Universität München

Reif R, Günthner WA (2009) Pick-by-vision: augmented reality supported order picking. The Visual Computer 25:461-467. doi:10.1007/s00371-009-0348-y

Reif R, Günthner WA, Schwerdtfeger B, Klinker G (2009) Pick-by-Vision Comes on Age: Evaluation of an Augmented Reality Supported Picking System in a Real Storage Environment. In: Spencer, SN (Ed) AFRIGRAPH '09: Proceedings of the 6th International Conference on Computer Graphics, Virtual Reality, Visualisation and Interaction in Africa. Association for Computing Machinery, New York

Schulte C (2009) Logistik. Verlag Franz Vahlen GmbH, München, 5., überarbeitete und erweiterte Auflage

Schwerdtfeger B, Reif R, Günthner WA, Klinker G, Hamacher D, Schega L (2009) Pickby-Vision: A first stress test. In Klinker G, Saito H, Höllerer T (Eds) 8th IEEE International Symposium on Mixed and Augmented Reality.

doi:10.1109/ISMAR.2009.5336484

Simões FM R V, Späth A (2018) ABILITY - Pick by voice in der Logistik, https://www.graesslin-kunststoffe.de/wp-content/uploads/sites/15/ppt_ABILITY.pdf, abgerufen am 07.07.2020

ten Hompel M, Schmidt T (2004) Warehouse Management. Springer, Berlin, 2. Auflage

ten Hompel M (2011) Warehouse Management. Springer, Berlin

ten Hompel M, Sadowsky V, Beck M (2011) Kommissionierung. Springer Verlag, Berlin Heidelberg

ten Hompel M, Heidenblut V (2008) Taschenlexikon Logistik. Springer Verlag, Berlin Heidelberg, 2. Auflage

Ternès A, Schieke S (2018) Mittelstand 4.0. Springer Fachmedien GmbH, Wiesbaden

Tompkins JA, White YA, Bozer EH, Tanchoco (2003) Facilities Planning. John Wiley and Sons, New Jersey 
Ubimax (2016) UbiMax. https://www.ubimax.com/web2017/de/news/wearables-in-thespotlight-de.html

Venkatesh V, Morris MG, Davis GB, Davis FD (2003) User Acceptance of Information Technology: Toward a Unified View. MIS Quarterly 27(3):425-478. doi: $10.2307 / 30036540$

Vuzix (2019) Vuzix Hardware. https://www.vuzix.com/, abgerufen am 07.07.2020

Webster J, Watson RT (2002) Analyzing the Past to Prepare for the Future: Writing a Literature Review. MIS Quarterly 26(2):xiii-xxiii

Wood J, Reiners T (2012) Gamification in Logistics and Supply Chain Education: Extending active learning. In: Kommers P, Issa T, Isaía P (Eds) IADIS International Conference on Internet Technologies \& Society. IADIS Press, Australia, Perth

Wölfle R, Schubert P (2006) Prozessexzellenz mit Business Software. Carl Hanser Verlag, München 